\title{
Arbor
}

\section{Sanidad y Democracia}

\author{
Carmen Martínez Aguayo, Manuel García Encabo
}

Arbor CLXX, 670 (Octubre 2001), 277-299 pp.

Con una clara intención analítica y reflexiva, intenta explicar las claves de la evolución de nuestro sistema sanitario, en donde se conjugan factores acaecidos fuera de nuestras fronteras, hasta nuestra propia maduración con la consiguiente afloración de exigencias que propician un desarrollo espectacular que va homologándose con otros países de nuestro entorno y que tienen su punto de arranque cuando nuestra Constitución reconoce el derecho a la salud de todos los ciudadanos.

\section{Del estado de beneficencia...}

El futuro de nuestro sistema sanitario es concebido, en muchos casos, como la consecuencia lógica de hechos y acontecimientos pasados o como producto de una ley que regiría de forma determinista la evolución de la sociedad y de la historia. Descubrir y seleccionar los hechos es darle sentido al pasado, pero si queremos proporcionar también una función social al presente es necesario vincular los hechos, la estructura y la organización a las distintas ideas, acciones y opciones humanas y políticas, tanto en el análisis del pasado y del presente como en el diseño e imagen del futuro (1).

En 1976 las diferencias en la comparación con los países de la Comunidad Europea del gasto dedicado a las principales funciones del Estado de Bienestar, eran suficientemente expresivas de la desatención en que se hallaban las necesidades y demandas de los ciu- 
dadanos: $34,8 \%$ en enseñanza, $77,6 \%$ en sanidad, $62,6 \%$ en seguridad social -pensiones y otras prestaciones sociales- y $39,7 \%$ en vivienda y desarrollo colectivo (2).

El Estado de Bienestar iniciado en 1883 en Alemania y acuñado, como tal, después de la segunda guerra mundial para definir las actuaciones del Estado relacionadas con la redistribución de las rentas, la prestación de los servicios de sanidad, educación y otros servicios de protección social como respuesta histórica a los problemas derivados de la industrialización cuando se rompe la protección de la familia y de la comunidad, no puede considerarse una realidad en nuestro país antes de 1975. La crisis del Estado de Bienestar no surge con la democracia, con la democracia surge el Estado de Bienestar (3).

Antes de la democracia los servicios sanitarios no pueden considerarse como un sistema organizado. La Ley de Bases de Sanidad Nacional (1944), vigente hasta 1986, respondía al concepto de un Estado liberal y benefactor, De acuerdo con ella las distintas administraciones públicas (Estado, Diputaciones, Ayuntamientos) debían ocuparse de la Salud Pública, dejando al individuo la responsabilidad de la atención a su enfermedad. La beneficencia se encargaba de atender las necesidades de la población sin recursos económicos. Sólo en el caso de algunas enfermedades consideradas de especial trascendencia social (tuberculosis, lepra y dermatosis, paludismo, cáncer, enfermedades sexuales, mentales.....) las administraciones públicas se encargaban de su asistencia a través de la red de hospitales y dispensarios (4).

Sólo con la idea de mejorar la productividad de las empresas mediante la reparación de la salud de los trabajadores, no el de contribuir a paliar las desigualdades y de reconocer el derecho a la salud, se crea en 1942 el Seguro Obligatorio de Enfermedad, financiado con las cuotas de los afiliados y sin ninguna transferencia ni implicación del Estado, dentro del sistema de protección social del Instituto Nacional de Previsión.

\section{...al estado de bienestar: La salud como derecho}

Las demandas de una sociedad que no se correspondía con la organización del Estado, la necesidad de modernización, no en el concepto más en boga de la doctrina neoliberal, sino en el sentido de aportar a cada individuo un máximo de libertad, de igualdad de oportunidades y de solidaridad (5), la necesidad, en resumen, de legitimar el nuevo 
sistema democrático impulsó un crecimiento de los servicios y del gasto público que reforzaban la función redistributiva del Estado (Tabla 1).

El reconocimiento del derecho a la salud de todos los ciudadanos, recogido en el artículo 43 de la Constitución de 1978 y la creación del Sistema Nacional de Salud en 1986, a través de la Ley General de Sanidad, supone un cambio radical sobre la situación precedente, los elementos nucleares sobre los que se asientan la asistencia garantizan el acceso universal y la equidad de los servicios:

- Financiación pública a través de los ingresos del Estado y mayoritariamente mediante impuestos.

Tabla 1

Clasificación funcional del gasto público (en \% del PIB)

\begin{tabular}{|l|c|c|c|}
\hline & 1975 & 1985 & 1995 \\
\hline Defensa Nacional & 6,60 & 6,75 & 6,30 \\
\hline Servicios Generales & 1,74 & 1,99 & 1,08 \\
\hline GASTOS COMUNES & $\mathbf{4 , 6 0}$ & $\mathbf{6 , 7 5}$ & $\mathbf{6 , 3 0}$ \\
\hline Pensiones & 5,62 & 10,21 & 11,63 \\
\hline Desempleo & 0,48 & 2,78 & 2,59 \\
\hline Otras Prestaciones & 3,15 & 1,34 & 1,77 \\
\hline Prestaciones Sociales & 9,25 & 14,33 & 15,99 \\
\hline Educación & 2,09 & 3,75 & 4,32 \\
\hline Sanidad & 3,77 & 4,67 & 5,92 \\
\hline Vivienda y servicios colectivos & 1,04 & 2,01 & 1,52 \\
\hline Bienes Sociales redistributivos & 6,90 & 10,43 & 11,76 \\
\hline GASTOS DE DISTRIBUCION & $\mathbf{1 6 , 1 5}$ & $\mathbf{2 4 , 7 6}$ & $\mathbf{2 7 , 7 5}$ \\
\hline Subvenciones de explotaciones & 1,14 & 2,44 & 1,77 \\
\hline Inversiones & 2,72 & 3,70 & 3,71 \\
\hline Transferencias del Capital & 1,01 & 2,77 & 1,87 \\
\hline GASTOS INTERVENCION ECONOMICA & $\mathbf{4 , 8 7}$ & $\mathbf{8 , 4 1}$ & $\mathbf{7 , 3 9}$ \\
\hline INTERESES DEUDA PUBLICA & $\mathbf{0 , 4 7}$ & $\mathbf{3 , 4 4}$ & $\mathbf{5 , 3 7}$ \\
\hline AJUSTES CLASIFICACION & $\mathbf{0}$ & $\mathbf{- 0 , 7 7}$ & $\mathbf{0 , 4 7}$ \\
\hline GASTOS TOTALES & $\mathbf{2 6 , 0 9}$ & $\mathbf{4 2 , 5 9}$ & $\mathbf{4 7 , 2 4}$ \\
\hline INGRESOS PUBLICOS & $\mathbf{2 5 , 7 2}$ & $\mathbf{3 5 , 6 5}$ & $\mathbf{4 0 , 5 9}$ \\
\hline DEFICIT PUBLICO & $\mathbf{- 0 , 3 5}$ & $\mathbf{- 6 , 9 3}$ & $\mathbf{- 6 , 5 5}$ \\
\hline
\end{tabular}

Fuente: Ortún. V. "Sistema Sanitario y Estado de Bienestar: problemas a la vista». Informe SESPAS 1998, según estimación de la Fundación FIES de las Cajas de Ahorro Confederadas. En Papeles de Economía Española nº8. pág. 17. 
Carmen Martínez Aguado, Manuel García Encabo

- Aseguramiento público sin entidades intermedias.

- Universalización de la cobertura y de las prestaciones del sistema.

- El acceso y las prestaciones sanitarias se realizaran en condiciones de igualdad efectiva.

- Provisión pública mayoritaria, considerando subsidiario el concurso de servicios privados a través de conciertos u otros sistemas contractuales.

- Integración de las políticas de promoción de la salud y de prevención de la enfermedad, junto a las prestaciones asistenciales.

- La política de salud estará orientada a la superación de los desequilibrios territoriales y sociales.

La universalización, el avance tecnológico de una sociedad industrial que permite nuevas aplicaciones en los medios diagnósticos y terapéuticos y las nuevas demandas de una sociedad desarrollada han caracterizado la historia de las últimas décadas de nuestro Sistema Sanitario como una época de expansión y crecimiento, más significativo en la década de los años ochenta y de forma más controlada, en referencia a la evolución del PIBn, en los años noventa.

En 1977, a pesar de la incorporación de nuevos colectivos a la seguridad social, fundamentalmente en la etapa $1953-1962$, en forma de regímenes especiales, se estima que el sistema de seguridad social daba sólo cobertura al $77 \%$ de la población, este porcentaje ha ido creciendo de forma paulatina hasta llegar a la práctica universalización (Decreto sobre Universalización de 1989), lo que ha supuesto la incorporación de más de siete millones de personas. (Tabla 2).

En 1997 el gasto dedicado a la asistencia sanitaria representaba el $12,2 \%$ del conjunto de los presupuestos públicos, siendo superado únicamente por la cantidad dedicada a la percepción de pensiones. Durante el periodo 1984-1998 (Tabla 3), el gasto sanitario creció en nuestro país un 527,16\%, mientras que el PIBn, utilizado como referente para valorar el crecimiento del gasto sanitario, crecía únicamente el $217,21 \%$.

A pesar de este crecimiento, el porcentaje de gasto sanitario en relación con el PIB sigue siendo (7,3\%, 78,2 \% de gasto público) uno de los menores de la Europa occidental. En 1994 el gasto sanitario por ciudadano en términos de paridades de poder adquisitivo era de 1419 dólares USA para la media de los países de la Europa occidental, situándose España (1005 dólares USA) por delante únicamente de Portugal, Grecia y Turquía (6). 


\section{Sanidad y Democracia}

Tabla 2

Evolución de la cobertura sanitaria en España

\begin{tabular}{|c|c|c|c|c|c|}
\hline \multirow{2}{*}{ MODALIDADES } & \multirow{2}{*}{1987} & \multicolumn{2}{|c|}{1993} & \multicolumn{2}{|c|}{1995} \\
\hline & & Pobl. Adulta & Pobl Infantil & Pobl. Adulta & Pobl Infantil \\
\hline Cobertura Pública & 97,1 & 98,5 & 98,5 & 99,6 & 99,6 \\
\hline - Seguridad Social & 90,5 & 93,8 & 93,7 & 94,8 & 96,2 \\
\hline $\begin{array}{l}\text { - Mutuas del Estado } \\
\text { acogidas a SS } \\
\end{array}$ & 2,8 & 2,5 & 1,8 & 2,5 & 1,2 \\
\hline $\begin{array}{l}\text { - Mutuas del Estado } \\
\text { (seguro privado) }\end{array}$ & 3,0 & 2,0 & 2,7 & 2,2 & 2,1 \\
\hline - Beneficencia & 0,9 & 0,2 & 0,3 & 0,1 & 0,1 \\
\hline Seguro médico de privado & 5,6 & 6,4 & 5,9 & 9,3 & 8 \\
\hline Seguro médico de empresa & 1,8 & 1,5 & 0,6 & 1,8 & 1,3 \\
\hline Iguala médica & 1,8 & 0,1 & 0,2 & 0,1 & 0,2 \\
\hline Otros & 0,4 & 0,5 & 0,1 & 0,3 & 0,2 \\
\hline
\end{tabular}

Fuente: Fernández, R. Con datos de MSC e INE, Encuesta de Cobertura Sanitaria 1987. Encuesta Nacional de Salud 1993 y 1995. Población infantil: En 1993 y 1995 hasta 16 años. En 1987 hasta 18 años. La suma de coberturas es mayor del $100 \%$ al existir personas con cobertura doble.

Tabla 3

Evolución del gasto sanitario y del PIBn

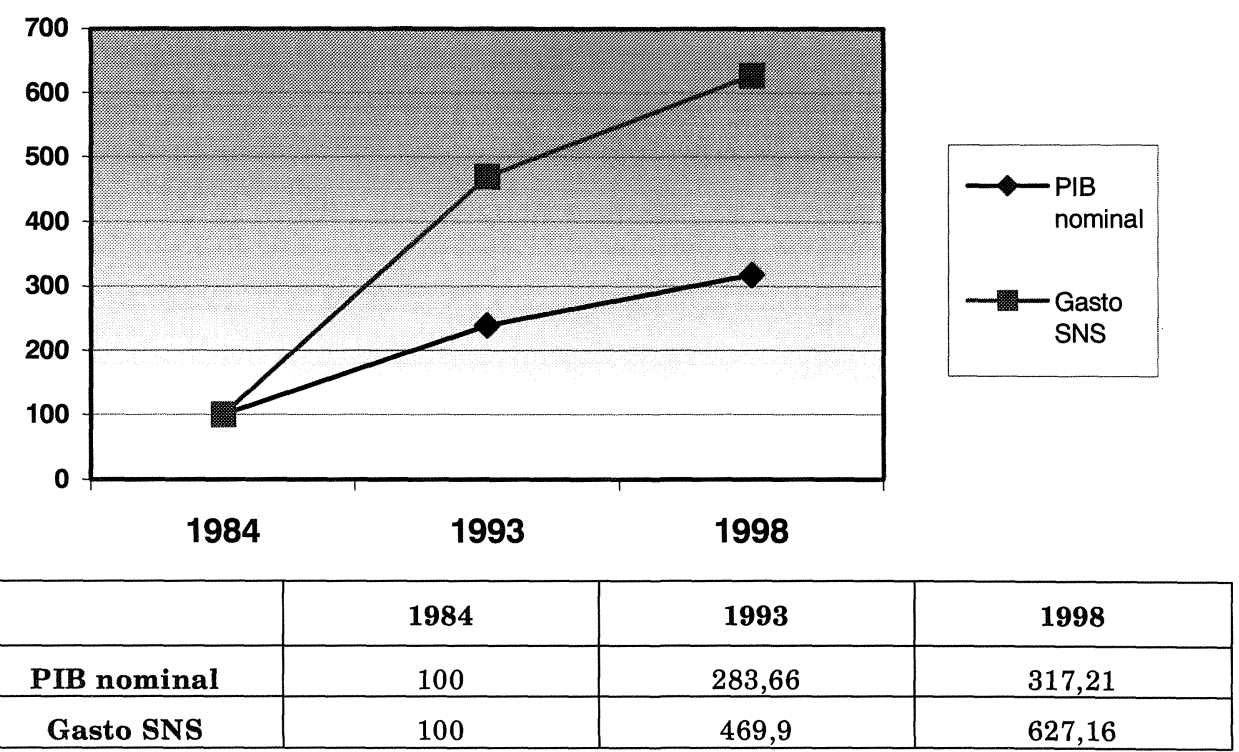

Fuente: «Escenarios de Financiación»(PESAS 1997-2000). Elaboración propia. 


\section{Una oferta de servicios adecuada}

El incremento y coordinación de la red asistencial mediante la integración de las distintas redes (Hospitales Universitarios, AISNA, Entidades Locales, etc.) y la puesta en funcionamiento de nuevos Centros en territorios con carencia de accesibilidad o de gran crecimiento demográfico, ha permitido la adecuación de la oferta a las características demográficas y socio-culturales de la población.

La evolución del número de camas para enfermos agudos es suficientemente significativa del esfuerzo realizado en la racionalización y adecuación de la oferta. Desde 1985 el número de camas para cada 1000 habitantes ha permanecido constante (2,4 camas/1000 habitantes) a pesar de haber entrado en funcionamiento un número significativo de hospitales. Además de la adecuación territorial habría que mencionar la cualificación y potencialidad de la oferta: La oferta total de camas ha descendido en un 10\% (1985-1994), pero de forma muy desigual según su utilidad, han aumentado hasta un $215 \%$ en hospitales geriátricos y de crónicos, se ha reducido de forma notable en hospitales infantiles $(77 \%)$, maternales (25\%) y psiquiátricos de larga duración (37\%), por el contrario se ha triplicado el número de camas psiquiátricas en los hospitales de agudos (7).

La reforma de los servicios de Atención Primaria ha significado una mejora notable en la relación con la oferta y la accesibilidad de los servicios. En los años setenta, la historia y la ciencia pasaban por la medicina general, la desprofesionalización, el carácter puramente administrativo de los servicios, el desprestigio social de los profesionales eran los signos más evidentes de su anacronismo, por el contrario, hoy los Centros de Salud son las instituciones mejor valoradas por los ciudadanos. Actualmente prestan servicios en la sanidad pública un médico general-pediatra por cada 1536 ciudadanos, habiendo ampliado más del $75 \%$ de los profesionales su oferta horaria de dos horas y media a siete horas al día, una red de Centros de Salud y Consultorios Locales se extiende por todo el territorio del país impidiendo cualquier problema de accesibilidad el tiempo medio de consulta, indicador que condiciona la calidad del servicio, se ha duplicado en los últimos años, sobrepasando los cinco minutos para consultas de carácter leve y los treinta minutos para casos programados y con una mayor complejidad.

La capitalización de los Centros e Instituciones sanitarias tanto en recursos humanos, como en tecnología y nuevas formas de oferta han sido otras de las características de estos años. Las remuneraciones de personal representaban en 1975 el 43,8\% del gasto y en 1990 el 


\section{Sanidad y Democracia}

$50,5 \%$ (8), entre 1985 y 1994 el número de profesionales contratados en los Hospitales públicos se incremento en un 36,16\% (7). El desarrollo del programa de formación MIR ha supuesto, con toda seguridad, la aportación más importante a la calidad de la asistencia, sustituyendo sistemas de formación no reglados ni acreditados a los que se tenía únicamente acceso desde criterios discrecionales. La creación de la especialidad de Medicina Familiar y Comunitaria junto al desarrollo de una organización que favorece el trabajo en equipo ha sacado a la Atención Primaria del estado acientífico en el que se encontraba, incorporándola al conocimiento y al hacer de la medicina contemporánea.

Las nuevas tecnologías médicas, incuestionables por su aportación al diagnóstico y tratamiento, se han incorporado de forma progresiva $\mathrm{y}$ accesible en todo el territorio.

Tabla 4

Equipos de alta tecnología disponible por CCAA

\begin{tabular}{|l|c|c|c|c|c|c|c|c|}
\hline \multicolumn{1}{|c|}{ Comunidad Autónoma } & TAC & RM & GAM & HEM & ASD & LIT & BCO & ALI \\
\hline ANDALUCÍA & 59 & 15 & 24 & 21 & 17 & 9 & 11 & 8 \\
\hline ARAGÓN & 10 & 3 & 0 & 4 & 4 & 2 & 2 & 1 \\
\hline PPDO. DE ASTURIAS & 12 & 4 & 4 & 2 & 2 & 1 & 3 & 1 \\
\hline ILLES BALEARS & 15 & 5 & 6 & 2 & 3 & 3 & 1 & 2 \\
\hline CANARIAS & 23 & 4 & 7 & 7 & 7 & 5 & 4 & 2 \\
\hline CANTABRIA & 3 & 1 & 0 & 2 & 1 & 0 & 2 & 1 \\
\hline CASTILLA-LA MANCHA & 13 & 3 & 4 & 2 & 3 & 3 & 1 & 0 \\
\hline CASTILLA Y LEÓN & 21 & 5 & 5 & 4 & 6 & 3 & 5 & 2 \\
\hline CATALUÑA & 44 & 19 & 9 & 31 & 20 & 13 & 15 & 9 \\
\hline COMUNIDAD VALENCIANA & 32 & 10 & 10 & 8 & 11 & 7 & 5 & 4 \\
\hline EXTREMADURA & 8 & 1 & 0 & 1 & 2 & 0 & 2 & 1 \\
\hline GALICIA & 32 & 12 & 6 & 8 & 15 & 7 & 5 & 1 \\
\hline MADRID & 51 & 30 & 18 & 31 & 25 & 12 & 11 & 15 \\
\hline REGIÓN DE MURCIA & 11 & 4 & 0 & 3 & 4 & 1 & 1 & 1 \\
\hline C. FORAL DE NAVARRA & 6 & 3 & 4 & 1 & 2 & 2 & 1 & 3 \\
\hline PAÍS VASCO & 24 & 11 & 0 & 10 & 10 & 2 & 4 & 4 \\
\hline LA RIOJA & 2 & 0 & 0 & 0 & 0 & 0 & 1 & 0 \\
\hline CEUTA & 1 & 0 & 0 & 0 & 0 & 0 & 0 & 0 \\
\hline MELILLA & 0 & 0 & 0 & 0 & 0 & 0 & 0 & 0 \\
\hline TOTAL & 367 & 130 & 97 & 137 & 132 & 71 & 74 & 55 \\
\hline
\end{tabular}

Abreviaturas: TAC: Tomografía Axial Computerizada. RM: Resonancia Nuclear Magnética. GAM: Gammacámara (incluye SPECT). HEM: Sala de Hemodinámica. ASD: Angiagrafía por Sustracción Digital. LIT: Litroticia. BCO: Bomba de Cobalto. ALI: Acelerador de Partículas.

Fuente: MSC. «Catálogo de Hospitales. 1997» Información obtenida a través de Internet en el "web» del MSC, http//www.msc.es/. 
Tabla 5

Evolución de la actividad de consultas de Atención Especializada en el Servicio Andaluz de Salud. (1990-1997)

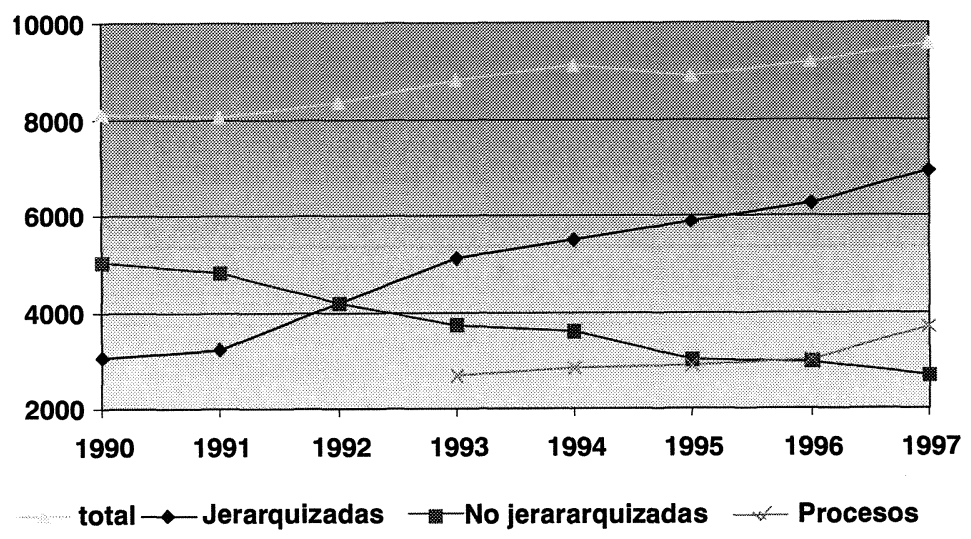

Fuente: Elaboración propia a partir de datos recogidos en las memorias del SAS. 1990-1997.

El incremento de la atención ambulatoria ha modificado de forma notable la oferta de servicios de los Hospitales, introduciendo modalidades asistenciales más adaptadas a las demandas de los usuarios y más eficientes para la organización. En el Servicio Andaluz de Salud, por ejemplo, el número de procesos atendidos en consultas externas en 1997 fue 3,70 millones, un millón mas que en 1993, el porcentaje de intervenciones quirúrgicas programadas realizadas ambulatoriamente alcanzó el 21,75\% y 185005 procesos fueron atendidos en hospital de día (10) (tabla 5). En el conjunto del sistema en 1985 se realizaron cerca de 21 millones de consultas, creciendo en 1994 hasta más de 39 millones (7).

Cambios profundos se han producido también en la oferta de servicios relacionada con los problemas de Salud Mental. Desde la creación de la Comisión Ministerial para la Reforma Psiquiátrica, en 1983, se ha ido produciendo una progresiva desinstitucionalización de estos enfermos, creándose unidades de agudos en los Hospitales Generales y unidades ambulatorias para la atención de estos enfermos en su comunidad. Desde 1985 se ha reducido en más de un $32 \%$ el número de camas psiquiátricas, ubicándose más de 2500 camas en hospitales generales y existiendo una Unidad de Salud Mental ambulatoria por cada 75000 habitantes (7).

\section{Cambios en la gestión y la organización}

Paralelamente a la adecuación y modernización de los servicios, se ha venido desarrollando importantes cambios en gestión y organi- 


\section{Sanidad y Democracia}

zación. El hecho más relevante y específico, sin duda, de nuestro Sistema Sanitario, acorde con la organización política y administrativa del Estado, ha sido el proceso de transferencias de competencias en materia de asistencia sanitaria a las Comunidades Autónomas. Esta decisión, sobre todo una vez que se finalize el proceso de transferencias, sitúa la responsabilidad política y administrativa más cerca del ciudadano a la vez que exige una colaboración y cooperación por parte de todos los responsables y todas las Administraciones, fragmenta el volumen de gestión permitiendo un mayor control y eficiencia de los recursos y facilita adecuar los servicios y la organización de los servicios a las características sociales y culturales de cada comunidad.

El crecimiento de la actividad y de los recursos utilizados ha exigido transformar y potenciar de forma radical la gestión y la dirección de los Centros e Instituciones sanitarias, pasando de procesos de administración artesanales, realizados por los propios profesionales y ligados directamente a la producción, a sistemas de gestión que incorporan, adecuándolos, procedimientos utilizados en la empresa privada y enfocados de forma primordial a la gestión económica y de personal. Aspectos, éstos considerados claves y sobre los que han girado las preocupaciones y la búsqueda de soluciones durante las últimas décadas.

En los hospitales públicos (8) el número de directivos por hospital ha crecido en un 8,92\% entre 1973 y 1990, el personal de carácter administrativo ha aumentado también en este mismo periodo en un 10,30\%. En Atención Primaria se ha pasado de una dirección ejercida por un inspector médico y una enfermera jefe por ambulatorio o consultorio, a un equipo de dirección muy similar al de los Hospitales (tabla 6).

Tabla 6

Evolución del número de personal de Dirección y administración en Hospitales Públicos

\begin{tabular}{|l|c|c|c|c|}
\hline & $\mathbf{1 9 7 3}$ & $\mathbf{1 9 8 3}$ & $\mathbf{1 9 9 0}$ & $\mathbf{7 3 / 9 0}$ \\
\hline Directivos por Hospital & $\mathbf{1 , 7 1}$ & 2,51 & 7,31 & $8,92 \%$ \\
\hline Empleados por Directivo & 133 & 219 & 110 & $-1,08 \%$ \\
\hline Ingresos por directivo & 1986 & 2326 & 1038 & $-3,74 \%$ \\
\hline Administrativos por Hospital & 12 & 36 & 62 & $10,30 \%$ \\
\hline Ingresos por Administrativo & 290 & 162 & 123 & $-4,95 \%$ \\
\hline
\end{tabular}

Fuente: Arruñada, B. Con datos del INE, Estadísticas de establecimientos sanitarios en régimen de internado, varios años. 
Carmen Martínez Aguado, Manuel García Encabo

Coincidiendo con decisiones adoptadas en otros países de nuestro entorno, se constituye en 1990 una Comisión para el Análisis y Evaluación del Sistema Nacional de Salud. En la argumentación por la que se justifica su constitución se reflejan aquellos razonamientos ya utilizados por países con servicios públicos consolidados y asentados y que contemplan, según las ideas predominantes en ese momento, la reforma del Estado de Bienestar siguiendo los cambios propugnados en el Reino Unido por gobiernos conservadores: «Para resolver las crecientes tensiones económicas, las profundas mutaciones demográficas, las nuevas actitudes sociales y la presión del progreso técnico-médico que demandan la transformación de los actuales sistemas sanitarios debería valorarse aquellos aspectos de «regulación versus competencia» (11).

En su informe, acorde también con las propuestas realizadas en otros países, la Comisión propone, entre otras, las siguientes recomendaciones (12):

- Separación de las funciones de financiación, aseguramiento y provisión.

- Transformación de los hospitales y otros centros de servicios sanitarios en sociedades estatales sometidas al derecho privado.

- Descentralización de la contratación de personal por cada uno de los Centros.

- Extender las prestaciones realizadas por entidades colaboradoras y mutuas.

- Desarrollar sistemas de co-pago para algunas prestaciones.

- Separar la financiación dedicada a actividades de promoción y prevención.

Estas recomendaciones, nunca asumidas oficialmente por ninguna de las Administraciones sanitarias, consonantes con el pensamiento dominante han impregnado el debate, los deseos y actuaciones durante la década de los noventa

Hasta ahora lo que se ha venido realizando son orientaciones que pueden incluirse en la llamada «competencia gestionada» a través de sistemas «híbridos de contratación» que se sitúan entre los procedimientos de mercado y jerarquizados y separan teóricamente la financiación, compra y provisión de servicios. Se parte de jerarquías públicas centralizadas, con sistemas de provisión integrados verticalmente y no implican un modelo de organización sanitaria único, existiendo múltiples posibilidades de combinar incentivos, controles, transacciones de mercado y acuerdos de cooperación (13).

Los modelos híbridos permiten acuerdos de cooperación con incentivos superiores a la estructura jerárquica pero con una capacidad de 
coordinación superior a la factible a través del mercado. Los Contratos Programas y los cambios en la entidad jurídica de los Centros (Empresas Públicas, Fundaciones, Consorcios, etc.) pueden considerarse variantes de esta alternativa (Tabla 7). El objetivo es transformar organizaciones basadas en los principios de jerarquización, centralización, control por normas (burocracias), en otras caracterizadas por acuerdos, control por incentivos, distribución de riesgos y de derechos de decisión.

Los cambios en la entidad jurídica de los Centros se han producido, hasta ahora, salvo en Cataluña, que ya partía de esta tradición, en la zona periférica del sistema (Centros nuevos, Unidades Específicas de Tecnología, Servicios de Emergencias.......), utilizándose para el resto y gran mayoría de los Centros Contratos Programas que establecen la producción y la financiación máxima disponible, con objetivos relacionados con la calidad y las mejoras en gestión.

Entre otros objetivos, los Contratos Programas pretenden favorecer los siguientes aspectos:

- Favorecer la política de salud de los distintos Sistemas y Servicios Sanitarios.

- Favorecer los cambios en gestión y organización establecidos por los distintos Sistemas y Servicios Sanitarios.

- Vincular la financiación a la actividad realizada y/o a los pacientes atendidos.

Tabla 7

Modelos de Contratos Sanitarios en la Sanidad Pública

\begin{tabular}{|l|l|l|}
\hline \multicolumn{1}{|c|}{ Tipo de contrato } & \multicolumn{1}{|c|}{ Características } & \multicolumn{1}{c|}{ Ejemplo } \\
\hline $\begin{array}{l}\text { Contrato programa } \\
\text { simulado o sombra }\end{array}$ & $\begin{array}{l}\text { Acuerdo de objetivos con finan- } \\
\text { ciación presupuestaria. Riesgo } \\
\text { cambio de directivos. }\end{array}$ & $\begin{array}{l}\text { Contrato programa INSALUD } \\
(1990-1996)\end{array}$ \\
\hline $\begin{array}{l}\text { Contrato programa } \\
\text { de Incentivos }\end{array}$ & $\begin{array}{l}\text { Igual que el anterior. Además in- } \\
\text { centivos para directivos y traba- } \\
\text { jadores, reversión de ingresos } \\
\text { para inversiones. }\end{array}$ & $\begin{array}{l}\text { Contrato Programa SAS } \\
\text { (1994 - 1996) }\end{array}$ \\
\hline $\begin{array}{l}\text { Contrato Programa } \\
\text { de riesgo parcial }\end{array}$ & $\begin{array}{l}\text { Subsidio público a una cuenta de } \\
\text { explotación. No hay riesgo de } \\
\text { quiebra, si ajustes laborales y mo- } \\
\text { dificaciones de las condiciones la- } \\
\text { borales. }\end{array}$ & $\begin{array}{l}\text { Contrato Programa con empresas } \\
\text { públicas }\end{array}$ \\
\hline Contrato externo & $\begin{array}{l}\text { Acuerdo entre entidades inde- } \\
\text { pendientes. }\end{array}$ & $\begin{array}{l}\text { Subcontrato de servicios no asis- } \\
\text { tenciales. }\end{array}$ \\
\hline
\end{tabular}

Fuente: Fernandez Diaz, J.M. (1996), "Modelos de contratación”, en BENGOA, R. (dir.). La sanidad: un sector en cambio. Un nuevo compromiso entre la Administración, usuarios y proveedores. Barcelona. SG editores. Pág. 77-86. 
Carmen Martínez Aguado, Manuel García Encabo

- Establecer tarifas homogéneas para actividad y procesos que favorezcan la disminución de la variabilidad clínica.

- Incentivar a los proveedores más eficientes y penalizar a los ineficientes.

- Mejorar y hacer transparente la información relativa a actividad, costes, productividad, etc.

Uno de los objetivos principales que se buscan en los Contrato Programa es variar la financiación retrospectiva e histórica de los Centros por un modelo prospectivo basado en la producción pactada y en las tarifas o precios de la misma. Para ello se han desarrollado y aplicado sistemas de información que cuantifican y cualifican la producción (UPA, GDR, Producto en Atención Primaria) (14) y de contabilidad analítica (SIGNO, COAN, ICAP) (15) que permiten establecer «cuentas de resultados» e incorporar el concepto de subvención o subfinanciación a la explotación en virtud de la diferencia entre ingresos y gastos.

La posibilidad de nuevas formas jurídicas y de organización para los Centros, testimoniales hasta el momento y sin incidencia real en el conjunto del Sistema, podrían generalizare y extenderse a partir de nuevas regulaciones realizadas por el gobierno del Partido Popular en la Ley de Habilitación de Nuevas Formas de Gestión (Ley 15/97) y en la nueva ordenación de los organismos públicos (Ley 6/97) y por las Comunidades Autónomas gobernadas por partidos nacionalistas y con servicios de salud transferidos (País Vasco: Ley 8/97, Cataluña: Ley 15/90 y 11/95......). Estas medidas, cuyo objetivo inicial es modificar de forma sustancial la gestión de los servicios sanitarios, van a permitir a medio plazo cambios sustanciales en características tan emblemáticas de nuestro sistema como la financiación y el aseguramiento único.

\section{Problemas a valorar}

Aunque, en la mayoría de las ocasiones, las propuestas de reforma se amparan en razonamientos técnicos y objetivos resulta evidente que la enumeración de los problemas, su magnitud, evolución, motivos y soluciones se ven influenciados y justificados por los valores e intereses del observador.

El crecimiento del gasto, la incorporación acelerada de nuevas tecnologías, el aumento y cualificación de la demanda debido a cambios demográficos y de nivel socio-económico y el bajo rendimiento de los servicios públicos son los problemas, que de una forma u otra, se 
citan como amenazas a la viabilidad del sistema sanitario y del Estado de Bienestar en su conjunto.

A pesar de ponerse en entredicho de forma sistemática el rendimiento de los hospitales públicos, su productividad se viene incrementando año tras año, el volumen de altas hospitalarias ha experimentádo en el período 1985-1994 un incremento cercano al 17\%. Paralelamente, la estancia media ha descendido un 21,5\%, pasando de 13,4 días de estancia por enfermo a 10,5. El índice de rotación ha pasado de 20 pacientes atendidos por cada cama en 1985 a casi 27 en 1994 (Tabla 8). El número de ingresos por médico, a pesar del incremento de dedicación horaria a las demandas en régimen ambulatorio, creció en un $8,5 \%$ en el periodo $1983-1990$ (7).

Tabla 8

Evolución de la estancia media, número de camas y altas en hospitales públicos. (1985-1995)

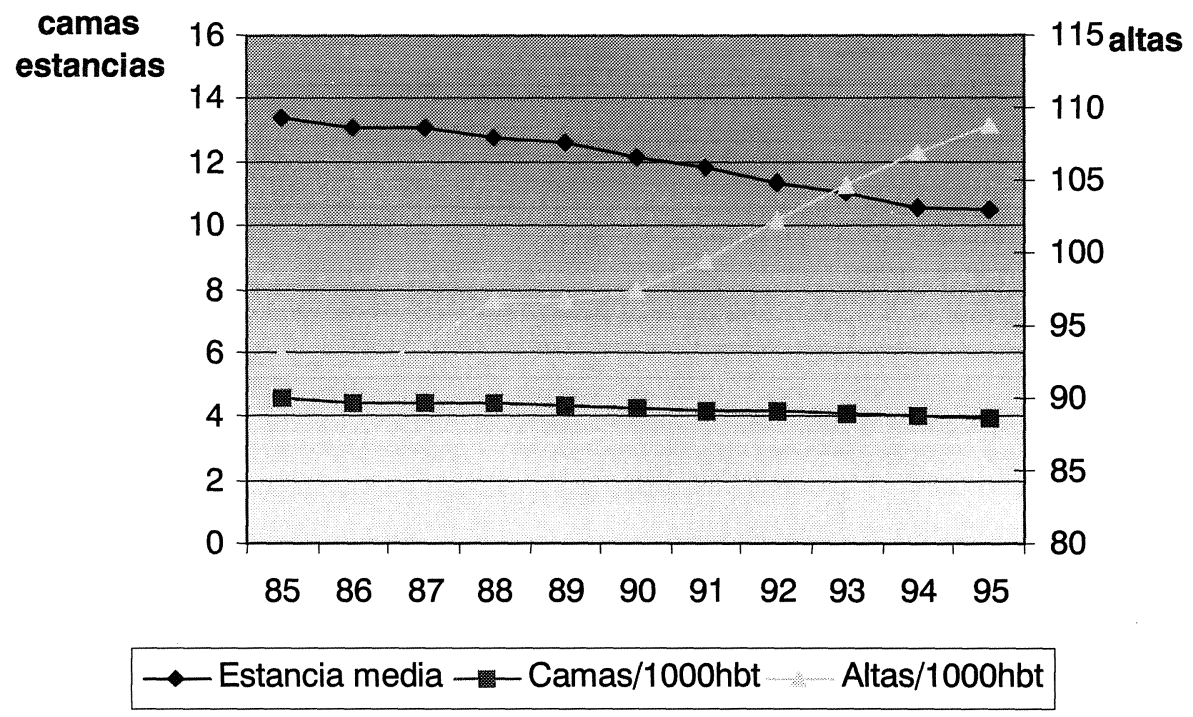

Fuente: INE. «España en cifras 1998: Salud» Información obtenida a través de Internet en el «web» del INE, http//www.ine.es/.

Aunque el crecimiento del gasto ha sido muy relevante durante las dos últimas décadas debido a la universalización del derecho a la asistencia y a la adecuación de la oferta de servicios, durante el período 1992-1996, coincidiendo con la implantación de un nuevo sistema de financiación de la asistencia sanitaria, acordado por todas las administraciones, los crecimientos interanuales disminuyeron en al menos 
algunos Servicios Regionales (SAS: 5,25; INSALUD: 5,82) por debajo de la evolución interanual del PIB nominal, parámetro propuesto para fijar el crecimiento de la financiación.

Si analizáramos someramente la evolución del gasto durante ese período, se advierte que únicamente aquellos ocasionados por la facturación de recetas ha puesto en riesgo el crecimiento pactado, al observar incrementos interanulaes por encima del $10 \%$, evolución que adquiere una mayor repercusión si se considera que el gasto por facturación de recetas se sitúa en torno al $30 \%$ del gasto total del sistema. Esta situación no se produjo tanto por una mayor prescripción de recetas sino, sobre todo, por el crecimiento del coste medio de la receta y por sustituciones de medicamentos o nuevas presentaciones con precios mucho más elevados.

A pesar del crecimiento del número de profesionales y su repercusión en la producción del gasto, no existe en los Centros sanitarios de nuestro sistema una inflación de recursos humanos sobre la situación en otros países europeos, por el contrario, hay un número significativamente menor en categorías profesionales como enfermería y personal administrativo.

El aumento de la demanda que se produjo en la década de los ochenta coincidió con la universalización de la asistencia sanitaria, la mejora de la oferta y el aumento del nivel de vida de los españoles. Durante los años noventa, del análisis de la demanda (frecuentación en Atención Primaria, ingresos hospitalarios, evolución de las listas de espera, etc.) no parece desprenderse que por parte de los ciudadanos se haya producido un incremento significativo de la misma, salvo en el caso de los servicios de urgencias.

El incremento de la demanda, achacada en muchos casos a la mala utilización que de los servicios realizan los ciudadanos o a la mala practica que realizan los profesionales, es, evidentemente, un elemento a valorar, pero sigue siendo inferior en nuestro país (atención primaria, estancias hospitalarias, número de prescripciones farmacéuticas) en comparación con la mayoría de países de nuestro entorno.

Las hipótesis sobre proyecciones demográficas prevén un incremento en la población mayor de 65 años, del $16,97 \%$ en el 2001, al $19,90 \%$ en el 2021 y del 30,21\% en el 2046 (16). Su incidencia sobre la demanda y gasto sanitario no debería realizarse de forma matemática sobre los actuales patrones de consumo, existen otras variables relacionadas con la organización de la sociedad, el nivel de renta, el estado de salud y de funcionalidad, el nivel cultural y de educación o las posibilidades de ocio, sobre las que también se pueden actuar y que condicionarán, en un sentido u otro, la demanda de cuidados sanitarios. 
Sin embargo, sí son previsibles modificaciones en el tipo de demanda, en parte inducidas por los propios profesionales, en parte por avances técnicos relacionados con mejoras en la oferta de servicios, y en parte también por las aspiraciones de los ciudadanos a una mayor calidad de vida y a una exigencia de más confort en su relación con las instituciones sanitarias.

Nuestro sistema sanitario ofrece unos niveles de eficiencia, como ha sido reconocido de forma repetida por informes y análisis de organismos internacionales, entre los más altos del mundo occidental, circunstancia que a veces se ignora cuando se destacan problemas y situaciones más graves y peor resueltas en algunos de los modelos que se proponen.

Posiblemente, los problemas más relevantes del Sistema Sanitario no tengan mucho que ver con los hasta ahora relacionados y que orientan a soluciones de reducción o aniquilación por inviabilidad económica, pero sí con la necesidad de huir del concepto schumpeteriano del estado y potenciar, por el contrario, el protagonismo de la sociedad y de los agentes implicados (17): Una mayor participación por parte de los ciudadanos y un mayor compromiso e identificación de los profesionales con la organización.

El crecimiento acelerado del sistema, la incorporación de nuevas formas de gestión que han incrementado los procedimientos administrativos y burocráticos, la necesidad de buscar inicialmente la eficiencia económica del sistema sobre otros resultados y la rigidez y centralización de las decisiones, ha ocasionado la escasa identificación de ciudadanos y profesionales con el sistema y un entorno que dificulta tanto las relaciones entre ellos como el control microeconómico del gasto.

El ciudadano, el usuario, ha intercambiado un incremento sustancial de su contribución económica (Tabla 9) a cambio de convertirse en sujeto pasivo de derechos, sin información suficiente para ejercer la elección de profesional o Centro y sobre todo sin participación adecuada para orientar y controlar unos servicios de los que es tanto usuario como propietario.

Por otra parte, la diferencia de objetivos entre dirección y profesionales médicos y la disminución de la influencia de éstos en la toma de decisiones ha ocasionado que la desconfianza, la frustración y la impotencia hayan sido las constantes en la relación entre organización y profesionales. Las soluciones siempre se han fiado a la modificación del tipo de relaciones laborales: funcionarización, laboralización, nuevo estatuto, han sido reclamados como soluciones definitivas pero nunca iniciadas. El profesional, el médico, ha sido percibido en demasiadas 


\section{Carmen Martínez Aguado, Manuel García Encabo}

ocasiones como enemigo de la organización, sin ser ni unos ni otros conscientes de que para la mayoría de los profesionales su trabajo en el Hospital o en el Centro de Salud es y va a ser su única fuente de ingresos y de desarrollo profesional a lo largo de toda su vida laboral.

Tabla 9

Impuesto comparados. En \% del PIB

\begin{tabular}{|l|c|c|c|}
\hline \multicolumn{1}{|c|}{ PAIS } & $\mathbf{1 9 7 0}$ & $\mathbf{1 9 8 0}$ & $\mathbf{1 9 9 5}$ \\
\hline Alemania & 22,8 & 24,8 & 23,6 \\
\hline Bélgica & 24,9 & 30,9 & 30,5 \\
\hline Dinamarca & 38,8 & 44,7 & 50,1 \\
\hline Finlandia & 29,6 & 29,8 & 33,4 \\
\hline Francia & 22,3 & 23,9 & 25,2 \\
\hline Grecia & 17,7 & 19,7 & 27,8 \\
\hline Irlanda & 28,5 & 29,0 & 30,3 \\
\hline Italia & 16,3 & 18,7 & 28,8 \\
\hline Japón & 15,3 & 18,0 & 18,1 \\
\hline Canadá & 28,3 & 28,3 & 30,0 \\
\hline Luxemburgo & 22,1 & 32,8 & 31,9 \\
\hline Holanda & 24,0 & 27,9 & 25,6 \\
\hline Noruega & 33,0 & 37,2 & 31,9 \\
\hline Australia & 26,6 & 28,4 & 36,8 \\
\hline Portugal & $\mathbf{1 5 , 4}$ & 17,8 & 25,3 \\
\hline Suecia & 33,8 & 34,8 & 35,8 \\
\hline Suiza & 18,2 & 21,3 & 21,4 \\
\hline España & $\mathbf{1 0 , 6}$ & $\mathbf{1 2 , 4}$ & $\mathbf{2 1 , 8}$ \\
\hline Reino Unido & $\mathbf{3 1 , 8}$ & 29,4 & 29,0 \\
\hline EEUU & 23,0 & 21,0 & 20,6 \\
\hline
\end{tabular}

Fuente: Lafontaine. O. (1998) con datos del Ministerio Alemán de Hacienda en «No hay que tener miedo a la globalización" Biblioteca Nueva. Madrid.

Este extrañamiento del núcleo profesional y productivo de la organización coincide paradójicamente con el incremento de las repercusiones que las decisiones médicas ocasionan. El incremento de la tecnología en medios diagnósticos y terapéuticos, la existencia de nuevas subespecialidades que complican el circuito de decisiones, la desfiguración del umbral entre enfermedad y malestar llevan consigo que de las decisiones profesionales dependan, más que nunca, los resultados 
en salud de los servicios y el coste económico de los mismos, siendo necesario transformar organizaciones que no han dejado de regirse por principios administrativos, en organizaciones de profesionales.

\section{Alternativas de reforma}

Actualmente, bajo la óptica de objetivos enunciados de forma similar, se proponen distintas alternativas de cambio y reforma general de los Sistemas y Servicios sanitarios. Estas reformas plantean actuaciones sobre aspectos y niveles de gestión muy diferentes. Para entender y explicar estas alternativas podríamos clasificar sus actuaciones estructurando la gestión de los servicios sanitarios en los tres siguientes niveles:

1. Macrogestión: Actuaciones relacionadas con la financiación, aseguramiento y derecho a las prestaciones.

2. Mesogestión: Actuaciones relacionadas con la entidad y gestión de Centros e Instituciones sanitarias.

3. Microgestión: Actuaciones que se sitúan en la relación con profesionales y usuarios.

Aunque, evidentemente, todo intento de clasificación supone simplificar, radicalizar las distintas alternativas y no contemplar situaciones mixtas, las propuestas existentes en el mercado de la gestión y la política pueden agruparse en dos grandes bloques. (Figura 1).

Aquellas que sitúan el núcleo de los cambios en la Microgestión: proponiendo una participación efectiva de los ciudadanos y modificando las relaciones con los profesionales al objeto de transferirles un mayor grado de autonomía y responsabilidad. O aquellas otras, que orientan las reformas hacia la Macrogestión y Mesogestión, creando mercados internos y externos más o menos regulados (18) (19) (20).

Las repercusiones reales de una y otra alternativa sobre la gestión de los centros y las relaciones con profesionales y ciudadanos inciden, a medio plazo, en organizaciones distintas y con realidades muy diferentes respecto a la equidad y accesibilidad de los servicios.

Una organización vertebrada sobre la microgestión, supondría situar en la dirección de los Centros (Hospitales, Direcciones de Atención Primaria) la responsabilidad de crear entornos que faciliten el trabajo de los profesionales y de asegurar los contenidos de regulación respecto 
a los derechos de los ciudadanos, promoviendo únicamente la externalización o cambio de entidad jurídica para aquellos servicios que no están directamente relacionados con la asistencia sanitaria y que pueden transferir riesgos reales a la iniciativa privada.

Las medidas o propuestas relacionadas con el ciudadano y el usuario no se incluyen, en este caso, en el campo de las transferencias de responsabilidad y riesgo a los distintos agentes del sistema; por el contrario, se trataría de considerar al ciudadano como portador de derechos, en su doble condición de financiador y usuario, al que se le debe proporcionar información, participación real y capacidad de elección, para que mediante su opinión y utilización condicione la oferta y gestión de los Centros.

Respecto a los profesionales, se incorpora, de forma progresiva, una mayor autonomía y responsabilidad a través de nuevas formas de gestión de personal y la constitución de unidades societarias que permitan relacionar consecuencias según resultados obtenidos en sus decisiones clínicas, dentro de la variabilidad y la incertidumbre de la práctica clínica, y la utilización de recursos y tecnología de los que son responsables.

Por el contrario, proponer de forma prioritaria modificaciones en la mesogestión se ha justificado por la necesidad de mejorar la gestión de personal y la eficiencia económica de los Centros mediante la reducción de los costes y el incremento de la actividad. Para ello se promueven cambios en la entidad jurídica de los Centros, introduciéndose nuevas formas públicas de gestión (empresas, consorcios, fundaciones) que huyen, hasta cierto punto, del derecho administrativo (intervención, compras, normas presupuestarias, endeudamiento, relaciones laborales), o estableciendo la provisión de los servicios a través de contratos con empresas privadas.

El usuario adquiere la condición de cliente, al que hay que captar para incrementar los ingresos financieros mediante técnicas de información y marketing; de la elección del usuario depende en parte la eficiencia económica de los Centros. Las relaciones con los profesionales se flexibilizan a efectos de contar con un recurso óptimo en la producción de beneficios para el Centro.

Una y otra alternativa suponen también distintas transacciones entre los Centros y el financiador. El cambio en la entidad jurídica de los Centros o la delegación de la provisión de servicios en entidades privadas, favorece, en teoría, establecer una auténtica función de compra, basada en el volumen y precio de la actividad y la definición clara de responsabilidades para cada uno de los agentes. Mantener 
el carácter administrativo de los Centros favorece, por el contrario, la corresponsabilidad de los resultados y la inclusión en los acuerdos de objetivos de salud y calidad al aportarse un menor riesgo a los proveedores.

Ninguna solución técnica o de organización dispone de bondades suficientes para ser útil en todos los objetivos, o ser la solución para la totalidad de los problemas. Mantener el carácter administrativo de los Centros y promover cambios en la relación con profesionales y usuarios puede hacer imposible, de no flexibilizarse ciertos procedimientos administrativos, una organización profesional orientada a la producción, la calidad y las relaciones satisfactorias con los usuarios, así como disponer de la capacidad de establecer consecuencias significativas en virtud de los resultados. La creación de mercados internos y externos, a través de cambios en la entidad jurídica de los Centros (Figura 2), puede ocasionar una disminución de la equidad y accesibilidad a los servicios, siendo necesario introducir para su control un alto nivel de regulación y de costes de transacción que anulen los efectos positivos del mercado.

Por lo tanto, el optar por una u otra organización, dependerá de las ideas u objetivos que cada Servicio o Sistema Sanitario considere más importante en su toma de decisiones: La salud como bien económico o la salud como derecho efectivo; la búsqueda de la eficiencia económica o de la eficiencia en virtud de la efectividad y calidad de los servicios; situar el núcleo básico de responsabilidad, con sus riesgos y beneficios, en los poseedores de las decisiones reales (profesionales sanitarios) o en los responsables formales de la organización (gestores).

Un proceso de rediseño organizativo cuyo objetivo sea situar a la gestión clínica en el núcleo de la organización e incorporar al profesional sanitario a la gestión de los recursos utilizados en su propia práctica clínica, obligaría, en cualquier caso, a definir las responsabilidades, organización y procedimientos de los distintos niveles de gestión, separando nítidamente las siguientes funciones (21):

- Función de gestión clínica

- Función de gestión de recursos

- Función reguladora

Función de gestión clínica: desarrollada en las Unidades Clínicas, lugares de encuentro entre los ciudadanos y la organización, nivel básico y principal de gestión, siendo referentes para toda la organización $\mathrm{y}$ priorizándose las recomendaciones y modificaciones que mayor incidencia tengan en su correcto funcionamiento: 
Figura 1

ALTERNATIVAS DE REFORMA DE LOS SERVICIOS SANITARIOS

\begin{tabular}{|c|c|}
\hline $\begin{array}{c}\text { SALUD: } \\
\text { Derecho efectivo }\end{array}$ & $\begin{array}{l}\text { SALUD: } \\
\text { Bien económico }\end{array}$ \\
\hline Procedimientos & Procedimientos \\
\hline $\begin{array}{l}\text { Relación con los usuarios } \\
\text { (Democratización) } \\
\text { Relación profesionales } \\
\text { (Descentralización) }\end{array}$ & $\begin{array}{c}\text { Creación de mercados } \\
\text { Internos } \\
\text { Externos }\end{array}$ \\
\hline
\end{tabular}

\begin{tabular}{|c|c|}
\hline \multicolumn{2}{|c|}{ MESOGESTION } \\
\hline \multicolumn{2}{|c|}{ Entidad de los Centros } \\
\hline $\begin{array}{c}\text { Administración } \\
\text { Externalización de los servicios ajenos a la } \\
\text { asistencia. }\end{array}$ & $\begin{array}{l}\text { Externalización mediante cambio de } \\
\text { entidad (competencia interna y externa } \\
\text { regulada) }\end{array}$ \\
\hline \multicolumn{2}{|c|}{ Objetivos } \\
\hline $\begin{array}{c}\text { De regulación } \\
\text { Entorno adecuado para la gestión clínica }\end{array}$ & $\begin{array}{l}\text { Eficiencia económica - beneficios. } \\
\text { Captación de clientes }\end{array}$ \\
\hline \multicolumn{2}{|c|}{ Relaciones con el financiador } \\
\hline $\begin{array}{c}\text { Acuerdo (equilibrio entre volumen, calidad } \\
\text { y satisfacción) }\end{array}$ & Compra (prioridad volumen, precio) \\
\hline
\end{tabular}

\begin{tabular}{|c|c|}
\hline \multicolumn{2}{|c|}{ WLROCESTION } \\
\hline \multicolumn{2}{|c|}{ Ciudadano - Usuario } \\
\hline $\begin{array}{l}\text { Participación } \\
\text { Información } \\
\text { Eleçión } \\
\text { Adecuación de la oferta } \\
\text { Adecuación de resultados }\end{array}$ & $\left.\right|^{\text {Cliente }}$ \\
\hline \multicolumn{2}{|c|}{ Profesionales } \\
\hline $\begin{array}{c}\text { Descentralización } \\
\text { Autonomía } \\
\text { Responsabilidad } \\
\text { Consecuencias según resultados (calidad, } \\
\text { efectividad, satisfacción usuarios, } \\
\text { económicos) }\end{array}$ & $\begin{array}{l}\text { Recurso que produce gasto } \\
\text { Consecuencias según resultados } \\
\text { (económicos) }\end{array}$ \\
\hline
\end{tabular}




\section{Sanidad y Democracia}
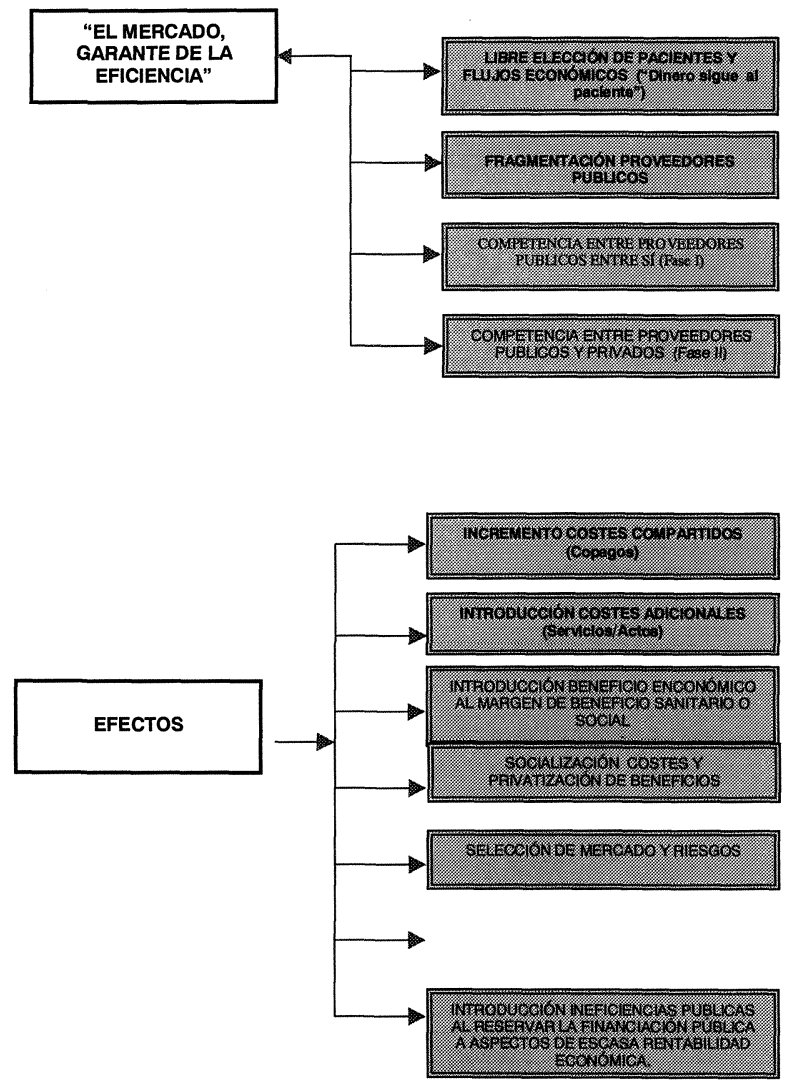

Fuente: Gavira L. El sistema sanitario público en Andalucía. Ponencia al Foro Andalucía, un nuevo siglo. Edición electrónica. Junta de Andalucía, Consejería de la Presidencia, 1998.

Configurándose las unidades como soportes fundamentales con cometidos establecidos, misiones específicas y con posibilidad de ser multidisciplinarias. Con líderes capaces de estimular e ilusionar.

Con un modelo de relación entre Centros y unidad capaz de orientar las actitudes y aptitudes, con capacidad de participar activamente en las decisiones y responsabilizándose de los resultados obtenidos, compartiendo los beneficios o pérdidas derivados de sus comportamientos (prestigio profesional y beneficio social). 
Estableciendo estrategias de avance según resultados previos de la Unidad. Introduciendo para aquellas Unidades con mejores resultados y mayores responsabilidades, fórmulas nuevas de relación entre Unidad y Centro.

Función de gestión de recursos: Ubicada fundamentalmente en la Dirección de Hospitales y de Atención Primaria, teniendo como objetivo básico el facilitar la actividad de las Unidades Clínicas, realizando $\mathrm{y}$ evaluando los acuerdos que se establezcan con ellas.

Conceptualizando las Direcciones de Hospitales y de Atención Primaria como un conjunto de instalaciones, procedimientos, servicios de apoyo y asesoramiento para un grupo de Unidades que pueden mantener distintas formas de relación, de «alquiler», con el Centro. Son una imagen o marca en la que se integran cada una de las Unidades Clínicas o «empresas», presentando una cuenta de resultados de la que dependerá su futuro o sus posibles adaptaciones.

Ejerciendo, además, la responsabilidad de que la asistencia se preste con criterios de equidad y accesibilidad y se respeten los derechos de los ciudadanos, a la vez que, como unidades de compra territorial de servicios, impiden la selección adversa de procesos y de grupos de pacientes.

Regidos por equipos directivos en los que se identifique claramente a los responsables de cada línea de producto, se refuercen las funciones de asesoramiento, formación, información e investigación, y en los que los profesionales integrados en las Unidades Clínicas tengan su representación, responsabilidad e influencia.

Función de regulación: Ejercida esencialmente por la Autoridad Sanitaria. Responsable de asegurar la equidad y accesibilidad, potenciando la orientación de la organización hacia resultados de efectividad y calidad de los servicios a través de su capacidad normativa y el correcto nivel técnico y de gestión en la implantación de las mismas.

Dotándose de un sistema de información coherente con la organización y el proceso de producción, que sea capaz de visualizar claramente las relaciones entre recursos, responsabilidad y resultados.

Separando de forma progresiva en la financiación de los Centros los ingresos por procesos o servicios de aquéllos condicionados por la estructura y ordenación de recursos, cuyos gastos no están relacionados con la gestión de los profesionales.

\section{Bibliografía}

1 Casanova, J. La historia social y los historiàdores. Editorial Crítica. Zaragoza 1991. 
2 Garcia Delgado, J.L. Serrano Sanz, J.M. Economía, en Tuñón de Lara, M. (dir) Historia de España: Transición y Democracia. Labor. Barcelona 1992.

3 ORTun, V. Sistema Sanitario y Estado de Bienestar: problemas a la vista. En Catalá, F., y De Manuel, E. (eds). Informe SESPAS 1998: La salud Pública y el futuro del Estado de Bienestar, Granada, Escuela Andaluza de Salud Pública, págs: 40-54. 1998.

4 Fernandez, Cuenca-Gomez, R., Análisis de los Servicios Sanitarios. En Catalá, F., y De Manuel, E. (eds). Informe SESPAS 1998: La salud Pública y el futuro del Estado de Bienestar, Granada, Escuela Andaluza de Salud Pública, págs, 251-298. 1998.

5 MARTIN Seco, J.F. Introducción en Lafontaine, O. y Müller, C. No hay que tener miedo a la globalización. Biblioteca Nueva. Madrid 1998.

6 OMS. Reforma Sanitaria en Europa. SAltman, B. Figueras, J.(dir). M.S.C. Madrid 1997.

7 Instituto NACIONAL DE EsTAdistica. Estadistica de Establecimientos Sanitarios con Régimen de Internado 1985-1994. Información obtenida a través de Internet en el "web" del INE, http//www.ine.es/. 1997.

8 ARruñadA, B., Bases para profesionalizar la Sanidad Pública, en López i Casanovas, G. y Rodríguez Palenzuela, D., (coord) (1997), La regulación de los servicios sanitarios en España, Madrid, Editorial Civitas, págs 157-212. 1997.

9 Ministerio DE SANIDAD Y CONSUMO Catálogo de Hospitales. 1997 Información obtenida a través de Internet en el «web» del MSC, http//www.msc.es/. 1997.

10 Servicio Andaluz De Salud. Memoria 1997. Consejería de Salud. Servicio Andaluz de Salud. Sevilla 1999.

11 Proposición Parlamentaria. Serie D. Número 13. Congreso de los Diputados. Enero. 1990.

12 Comision de Analisis y Evaluacion DEL Sistema Nacional de Salud. Informe $y$ Recomendaciones. Julio 1991.

13 Martín Martín, J. Manuel Kenoy, E. Reformas y cambios organizativos en el Sistema Nacional de Salud español. Papeles de Economía Española. 76. Pág. 176-191. 1998.

14 Servicio Andalud de SAlud Proyecto: Producto en Atención Primaria (Documento interno) 1999

15 SERvicio ANDAluZ DE SALUd Imputación de Costes en Atención Primaria (En prensa) 1998.

16 Fernandez Cordon. JA. Demografia, actividad y dependencia en España. Serie Economía Pública. Madrid. Fundación BBV. 1996.

17 CAmps. V. El malestar de la vida pública. Grijalbo. Barcelona. 1996.

18 Martin, Martin, J., de Manuel E., Carmona G., Martinez P. Los cambios necesarios para continuar la reforma sanitaria: eficiencia e incentivos en la Atención Primaria. $9^{a}$ Jornadas de Economía de la Salud. Barcelona, 1989.

19 SAltman RB, Von OtTer C. Revitalización de los sistemas de asistencia sanitaria pública: propuestas para la competencia pública en Suecia. Papeles de Gestión Sanitaria. Feb. 1988.

20 ENThoven AC. Reflections on the management of the National Health Service: an american looks at incentives to efficiency in Health Services mangement in the U.K. Nuffield Provincial Hospital Trust. London 1985

21 Servicio ANDALUZ DE SALUd, Plan Estratégico. Una propuesta de gestión pública diferenciada. Sevilla 1999. 